\title{
OSCILLATION CRITERIA FOR A CLASS OF PARTIAL FUNCTIONAL-DIFFERENTIAL EQUATIONS OF HIGHER ORDER
}

\author{
TARIEL KIGURADZE \\ Tbilisi I. Javakhishvili State University \\ Faculty of Physics \\ 3 Chavchavadze Ave., Tbilisi 380028 Georgia \\ TAKAŜI KUSANO \\ Fukuoka University \\ Department of Applied Mathematics, Faculty of Science \\ Fukuoka, 814-0180 Japan \\ NORIO YOSHIDA \\ Toyama University \\ Department of Mathematics, Faculty of Science \\ Toyama, 930-8555 Japan
}

(Received January, 2002; Revised May, 2002)

\begin{abstract}
Higher order partial differential equations with functional arguments including hyperbolic equations and beam equations are studied. Sufficient conditions are derived for every solution of certain boundary value problems to be oscillatory in a cylindrical domain. Our approach is to reduce the multi-dimensional oscillation problem to a one-dimensional problem for higher order functional differential inequalities.
\end{abstract}

Key words: Oscillation, Higher Order Partial Differential Equations, Functional Arguments.

AMS subject classifications: $35 \mathrm{~B} 05$.

\section{Introduction}

Oscillations of higher order partial differential equations have been developed by numerous authors. We refer the reader to Kiguradze and Stavroulakis [2], Liu and Fu [4], Onose and Yokoyama [5], Yoshida [10] for boundary value problems for higher order hyperbolic equations, and to Kusano and Yoshida [3], Travis and Yoshida [8] for characteristic initial value problems for higher order hyperbolic equations.

We are concerned with the oscillation of the higher order partial differential equation with functional arguments

$$
\frac{\partial^{N}}{\partial t^{N}}\left(u(x, t)+\sum_{i=1}^{\ell} h_{i}(t) u\left(x, \rho_{i}(t)\right)\right)
$$




$$
\begin{gathered}
-\left[\sum_{j=1}^{M}(-1)^{j-1} a_{j}(t) \Delta^{j} u(x, t)+\sum_{i=1 j=1}^{k} \sum_{j=1}^{M}(-1)^{j-1} b_{i j}(t) \Delta^{j} u\left(x, \tau_{i j}(t)\right)\right] \\
+c\left(x, t,\left(u\left(x, \sigma_{i}(t)\right)\right)_{i=1}^{m}\right)=f(x, t),(x, t) \in \Omega \equiv G \times(0, \infty),
\end{gathered}
$$

where $\Delta$ is the Laplacian in $\mathbb{R}^{n}$ and $G$ is a bounded domain in $\mathbb{R}^{n}$ with piecewise smooth boundary $\partial G$. It is assumed that:

$\left(\mathrm{H}_{1}\right) \quad h_{i}(t) \in C^{1}([0, \infty) ;[0, \infty))(i=1,2, \ldots, \ell)$, $a_{j}(t) \in C([0, \infty) ;[0, \infty))(j=1,2, \ldots, M)$, $b_{i j}(t) \in C([0, \infty) ;[0, \infty))(i=1,2, \ldots, k ; j=1,2, \ldots, M)$, and $f(x, t) \in C(\bar{\Omega}, \mathbb{R})$;

$\left(\mathrm{H}_{2}\right) \quad \rho_{i}(t) \in C^{1}([0, \infty) ; \mathbb{R}), \lim _{t \rightarrow \infty} \rho_{i}(t)=\infty(i=1,2, \ldots, \ell)$, $\tau_{i j}(t) \in C([0, \infty) ; \mathbb{R}), \lim _{t \rightarrow \infty} \tau_{i j}(t)=\infty(i=1,2, \ldots, k ; j=1,2, \ldots, M)$, $\sigma_{i}(t) \in C([0, \infty) ; \mathbb{R}), \lim _{t \rightarrow \infty} \sigma_{i}(t)=\infty(i=1,2, \ldots, m) ;$

$\left(\mathrm{H}_{3}\right) \quad c\left(x, t,\left(\xi_{i}\right)_{i=1}^{m}\right) \in C\left(\bar{\Omega} \times \mathbb{R}^{m} ; \mathbb{R}\right)$, $c\left(x, t,\left(\xi_{i}\right)_{i=1}^{m}\right) \geq \sum_{i=1}^{m} p_{i}(t) \varphi_{i}\left(\xi_{i}\right)$ for $\left(x, t,\left(\xi_{i}\right)_{i=1}^{m}\right) \in \Omega \times[0, \infty)^{m}$, $c\left(x, t,\left(-\xi_{i}\right)_{i=1}^{m}\right) \leq-\sum_{i=1}^{m} p_{i}(t) \varphi_{i}\left(\xi_{i}\right)$ for $\left(x, t,\left(\xi_{i}\right)_{i=1}^{m}\right) \in \Omega \times[0, \infty)^{m}$,

where $p_{i}(t) \in C([0, \infty) ;[0, \infty)), \varphi_{i}(\xi) \in C([0, \infty) ;[0, \infty))$, and $\varphi_{i}(\xi)$ are convex in $(0, \infty)$ $(i=1,2, \ldots, m)$.

The boundary conditions to be considered are the following:

(B $\left.\mathrm{B}_{1}\right) \quad \Delta^{j} u=\psi_{j+1}$ on $\partial G \times(0, \infty)(j=0,1, \ldots, M-1)$,

(B) $\quad \frac{\partial \Delta^{j} u}{\partial \nu}+(-1)^{j} \mu_{j+1} u=\widetilde{\psi}_{j+1}$ on $\partial G \times(0, \infty)(j=0,1, \ldots, M-1)$, where $\psi_{j+1}, \widetilde{\psi}_{j+1} \in C(\partial G \times(0, \infty) ; \mathbb{R}), \mu_{j+1} \in C(\partial G \times(0, \infty) ;[0, \infty))$ and $\nu$ denotes the unit exterior normal vector to $\partial G$.

Definition 1: By a solution of equation (1), we mean a function $u(x, t) \in C^{K}(\bar{G} \times$ $\left.\left[t_{-1}, \infty\right) ; \mathbb{R}\right) \cap C\left(\bar{G} \times\left[t_{-1}, \infty\right) ; \mathbb{R}\right)$ which satisfies (1), where $K=\max \{N, 2 M\}$,

$$
\begin{gathered}
t_{-1}=\min \left\{0 \min _{1 \leq i \leq \ell}\left\{\inf _{t \geq 0} \rho_{i}(t)\right\} \min _{\substack{1 \leq i \leq k \\
1 \leq j \leq M}}\left\{t \geq \inf _{i j} \tau_{i j}(t)\right\}\right\} \\
\bar{t}_{-1}=\min \left\{\min _{1 \leq i \leq m}\left\{\inf _{t \geq 0} \sigma_{i}(t)\right\}, 0\right\} .
\end{gathered}
$$

Definition 2: A solution $u$ of equation (1) is said to be oscillatory in $\Omega$ if $u$ has a zero in $G \times(t, \infty)$ for any $t>0$.

In the case where $N=2, M=1$, equation (1) can be reduced to the hyperbolic equation with functional arguments

$$
\begin{gathered}
\frac{\partial^{2}}{\partial t^{2}}\left(u(x, t)+\sum_{i=1}^{\ell} h_{i}(t) u\left(x, \rho_{i}(t)\right)\right) \\
-\left[a_{1}(t) \Delta u(x, t)+\sum_{i=1}^{k} b_{i 1}(t) \Delta u\left(x, \tau_{i 1}(t)\right)\right]
\end{gathered}
$$




$$
+c\left(x, t,\left(u\left(x, \sigma_{i}(t)\right)\right)_{i=1}^{m}\right)=f(x, t)
$$

whose oscillation was studied by Tanaka [7]. In case $N=M=2, h_{i}(t) \equiv 0$ and $b_{i j}(t) \equiv 0$, equation (1) can be reduced to the beam equation

$$
\frac{\partial^{2} u}{\partial t^{2}}-a_{1}(t) \frac{\partial^{2} u}{\partial x^{2}}+a_{2}(t) \frac{\partial^{4} u}{\partial x^{4}}+c\left(x, t,\left(u\left(x, \sigma_{i}(t)\right)\right)_{i=1}^{m}\right)=f(x, t),
$$

which was considered by Yoshida [9].

The purpose of this paper is to derive sufficient conditions for every solution of the boundary value problems for $(1),\left(B_{i}\right)(i=1,2)$ to be oscillatory in the cylindrical domain $\Omega=G \times(0, \infty)$. In Section 2 we reduce the oscillation problems for (1) to one-dimensional oscillation problems for higher order functional differential inequalities. In Section 3 we investigate the functional differential inequalities of higher order, and we obtain in Section 4 the oscillation criteria for (1) by combining the results of Sections 2 and 3.

\section{Reduction to One-Dimensional Oscillation Problems}

In this section we reduce the multi-dimensional oscillation problems for (1) to one-dimensional oscillation problems for functional differential inequalities of higher order.

We use the notation:

$$
\begin{gathered}
F(t)=\left(\int_{G} \Phi(x) d x\right)^{-1} \int_{G} f(x, t) \Phi(x) d x, \\
\Psi_{j}(t)=\left(\int_{G} \Phi(x) d x\right)^{-1} \int_{\partial G} \psi_{j} \frac{\partial \Phi}{\partial \nu}(x) d S,(j=1,2, \ldots, M), \\
\widetilde{F}(t)=\frac{1}{|G|} \int_{G} f(x, t) d x, \\
\widetilde{\Psi}_{j}(t)=\frac{1}{|G|} \int_{\partial G} \widetilde{\psi}_{j} d S,(j=1,2, \ldots, M),
\end{gathered}
$$

where $|G|=\int_{G} d x$ and $\Phi(x)$ is the eigenfunction of the eigenvalue problem

$$
\begin{gathered}
-\Delta w=\lambda w \text { in } G, \\
w=0 \text { on } \partial G
\end{gathered}
$$

corresponding to the first eigenvalue $\lambda_{1}>0$.

Theorem 1: Assume that $\left(H_{1}\right)-\left(H_{3}\right)$ hold. If the functional differential inequalities 


$$
\begin{gathered}
\quad \frac{d^{N}}{d t^{N}}\left(y(t)+\sum_{i=1}^{\ell} h_{i}(t) y\left(\rho_{i}(t)\right)\right)+\left(\sum_{j=1}^{M} \lambda_{1}^{j} a_{j}(t)\right) y(t) \\
+\sum_{i=1}^{k} \sum_{j=1}^{M} \lambda_{1}^{j} b_{i j}(t) y\left(\tau_{i j}(t)\right)+\sum_{i=1}^{m} p_{i}(t) \varphi_{i}\left(y\left(\sigma_{i}(t)\right)\right) \leq \pm G(t)
\end{gathered}
$$

have no eventually positive solutions, then every solution $u$ of the boundary value problem (1), $\left(B_{1}\right)$ is oscillatory in $\Omega$, where

$$
\begin{gathered}
G(t)=F(t)+\sum_{j=1}^{M} \sum_{p=0}^{j-1}(-1)^{j+p} \lambda_{1}^{p} a_{j}(t) \Psi_{j-p}(t) \\
+\sum_{i=1}^{k} \sum_{j=1}^{M} \sum_{p=0}^{j-1}(-1)^{j+p} \lambda_{1}^{p} b_{i j}(t) \Psi_{j-p}\left(\tau_{i j}(t)\right) .
\end{gathered}
$$

Proof: Suppose to the contrary that there exists a solution $u$ of the problem (1), ( $\left.\mathrm{B}_{1}\right)$ which is nonoscillatory in $\Omega$. First let $u>0$ in $G \times\left[t_{0}, \infty\right)$ for some $t_{0}>0$. The hypothesis $\left(\mathrm{H}_{3}\right)$ implies

$$
c\left(x, t,\left(u\left(x, \sigma_{i}(t)\right)\right)_{i=1}^{m}\right) \geq \sum_{i=1}^{m} p_{i}(t) \varphi_{i}\left(u\left(x, \sigma_{i}(t)\right)\right) \text { in } G \times\left[t_{1}, \infty\right)
$$

for some $t_{1} \geq t_{0}$. Hence (1) reduces to

$$
\begin{gathered}
\frac{\partial^{N}}{\partial t^{N}}\left(u(x, t)+\sum_{i=1}^{\ell} h_{i}(t) u\left(x, \rho_{i}(t)\right)\right) \\
-\left[\sum_{j=1}^{M}(-1)^{j-1} a_{j}(t) \Delta^{j} u(x, t)+\sum_{i=1}^{k} \sum_{j=1}^{M}(-1)^{j-1} b_{i j}(t) \Delta^{j} u\left(x, \tau_{i j}(t)\right)\right] \\
+\sum_{i=1}^{m} p_{i}(t) \varphi_{i}\left(u\left(x, \sigma_{i}(t)\right)\right) \leq f(x, t) \text { in } G \times\left[t_{1}, \infty\right) .
\end{gathered}
$$

Multiplying (3) by $\left(\int_{G} \Phi(x) d x\right)^{-1} \Phi(x)$ and then integrating over $G$, we obtain

$$
\frac{d^{N}}{d t^{N}}\left(U(t)+\sum_{i=1}^{\ell} h_{i}(t) U\left(\rho_{i}(t)\right)\right)
$$




$$
\begin{gathered}
-\left[\sum_{j=1}^{M}(-1)^{j-1} a_{j}(t) K_{\Phi} \int_{G} \Delta^{j} u(x, t) \Phi(x) d x\right. \\
\left.+\sum_{i=1}^{k} \sum_{j=1}^{M}(-1)^{j-1} b_{i j}(t) K_{\Phi} \int_{G} \Delta^{j} u\left(x, \tau_{i j}(t)\right) \Phi(x) d x\right] \\
+\sum_{i=1}^{m} p_{i}(t) K_{\Phi} \int_{G} \varphi_{i}\left(u\left(x, \sigma_{i}(t)\right)\right) \Phi(x) d x \leq F(t), t \geq t_{1},
\end{gathered}
$$

where $K_{\Phi}=\left(\int_{G} \Phi(x) d x\right)^{-1}$ and

$$
U(t)=\left(\int_{G} \Phi(x) d x\right)^{-1} \int_{G} u(x, t) \Phi(x) d x .
$$

The following identities hold:

$$
\begin{gathered}
\int_{G} \Delta^{j} u(x, t) \Phi(x) d x=-\sum_{p=0}^{j-1}\left(-\lambda_{1}\right)^{p} \int_{\partial G} \psi_{j-p} \frac{\partial \Phi}{\partial \nu}(x) d S \\
+\left(-\lambda_{1}\right)_{G}^{j} u(x, t) \Phi(x) d x, \quad(j=1,2, \ldots, M)
\end{gathered}
$$

(cf. Yoshida [10, Lemma 1]), and therefore we have

$$
\begin{gathered}
\sum_{j=1}^{M}(-1)^{j-1} a_{j}(t) K_{\Phi} \int_{G} \Delta^{j} u(x, t) \Phi(x) d x \\
=\sum_{j=1}^{M} \sum_{p=0}^{j-1}(-1)^{j+p} \lambda_{1}^{p} a_{j}(t) \Psi_{j-p}(t)-\sum_{j=1}^{M} \lambda_{1}^{j} a_{j}(t) U(t) .
\end{gathered}
$$

Analogously, we obtain

$$
\begin{gathered}
\sum_{i=1}^{k} \sum_{j=1}^{M}(-1)^{j-1} b_{i j}(t) K_{\Phi} \int_{G} \Delta^{j} u\left(x, \tau_{i j}(t)\right) \Phi(x) d x \\
=\sum_{i=1}^{k} \sum_{j=1}^{M} \sum_{p=0}^{j-1}(-1)^{j+p} \lambda_{1}^{p} b_{i j}(t) \Psi_{j-p}\left(\tau_{i j}(t)\right)-\sum_{i=1}^{k} \sum_{j=1}^{M} \lambda_{1}^{j} b_{i j}(t) U\left(\tau_{i j}(t)\right) .
\end{gathered}
$$


An application of Jensen's inequality yields

$$
K_{\Phi} \int_{G} \varphi_{i}\left(u\left(x, \sigma_{i}(t)\right)\right) \Phi(x) d x \geq \varphi_{i}\left(U\left(\sigma_{i}(t)\right)\right) .
$$

Combining (4)-(7), we see that $U(t)$ is an eventually positive solution of (2) with $+G(t)$. This is a contradiction. The case where $u<0$ in $G \times\left[t_{0}, \infty\right)$ can be treated similarly, and we conclude that $V(t) \equiv-U(t)$ is an eventually positive solution of (2) with $-G(t)$. This contradicts the hypothesis. The proof is complete.

Theorem 2: Assume that $\left(H_{1}\right)-\left(H_{3}\right)$ hold. If the functional differential inequalities

$$
\frac{d^{N}}{d t^{N}}\left(y(t)+\sum_{i=1}^{\ell} h_{i}(t) y\left(\rho_{i}(t)\right)\right)+\sum_{i=1}^{m} p_{i}(t) \varphi_{i}\left(y\left(\sigma_{i}(t)\right)\right) \leq \pm \widetilde{G}(t)
$$

have no eventually positive solutions, then every solution $u$ of the boundary value problem (1), $\left(B_{2}\right)$ is oscillatory in $\Omega$, where

$$
\widetilde{G}(t)=\widetilde{F}(t)+\sum_{j=1}^{M}(-1)^{j-1} a_{j}(t) \widetilde{\Psi}_{j}(t)+\sum_{i=1}^{k} \sum_{j=1}^{M}(-1)^{j-1} b_{i j}(t) \widetilde{\Psi}_{j}\left(\tau_{i j}(t)\right) .
$$

Proof: Suppose that there is a nonoscillatory solution $u$ of the problem (1), $\left(\mathrm{B}_{2}\right)$. Let $u>0$ in $G \times\left[t_{0}, \infty\right)$ for some $t_{0}>0$. Proceeding as in the proof of Theorem 1 , we see that (3) holds. Integrating (3) over $G$ and then dividing by $|G|$ yields

$$
\begin{gathered}
\frac{d^{N}}{d t^{N}}\left(\widetilde{U}(t)+\sum_{i=1}^{\ell} h_{i}(t) \widetilde{U}\left(\rho_{i}(t)\right)\right) \\
-\left[\sum_{j=1}^{M}(-1)^{j-1} a_{j}(t) \frac{1}{|G|} \int_{G} \Delta^{j} u(x, t) d x\right. \\
\left.+\sum_{i=1}^{k} \sum_{j=1}^{M}(-1)^{j-1} b_{i j}(t) \frac{1}{|G|} \int_{G} \Delta^{j} u\left(x, \tau_{i j}(t)\right) d x\right] \\
+\sum_{i=1}^{m} p_{i}(t) \frac{1}{|G|} \int_{G} \varphi_{i}\left(u\left(x, \sigma_{i}(t)\right)\right) d x \leq \widetilde{F}(t), t \geq t_{1}
\end{gathered}
$$

for some $t_{1} \geq t_{0}$, where

$$
\widetilde{U}(t)=\frac{1}{|G|} \int_{G} u(x, t) d x
$$


In view of the boundary condition $\left(\mathrm{B}_{2}\right)$, we have

$$
\begin{gathered}
\int_{G}(-1)^{j-1} \Delta^{j} u(x, t) d x=\int_{\partial G}(-1)^{j-1} \frac{\partial \Delta^{j-1} u}{\partial \nu} d S \\
=\int_{\partial G}(-1)^{j-1}\left(-(-1)^{j-1} \mu_{j} u+\widetilde{\psi}_{j}\right) d S \\
=\int_{\partial G}\left(-\mu_{j} u+(-1)^{j-1} \widetilde{\psi}_{j}\right) d S \\
\leq|G|(-1)^{j-1} \widetilde{\Psi}_{j}(t), \quad(j=1,2, \ldots, M)
\end{gathered}
$$

and therefore,

$$
\sum_{j=1}^{M}(-1)^{j-1} a_{j}(t) \frac{1}{|G|} \int_{G} \Delta^{j} u(x, t) d x \leq \sum_{j=1}^{M}(-1)^{j-1} a_{j}(t) \widetilde{\Psi}_{j}(t) .
$$

Similarly, we obtain

$$
\begin{gathered}
\sum_{i=1}^{k} \sum_{j=1}^{M}(-1)^{j-1} b_{i j}(t) \frac{1}{|G|} \int_{G} \Delta^{j} u\left(x, \tau_{i j}(t)\right) d x \\
\quad \leq \sum_{i=1}^{k} \sum_{j=1}^{M}(-1)^{j-1} b_{i j}(t) \widetilde{\Psi}_{j}\left(\tau_{i j}(t)\right) .
\end{gathered}
$$

An application of Jensen's inequality shows that

$$
\frac{1}{|G|} \int_{G} \varphi_{i}\left(u\left(x, \sigma_{i}(t)\right)\right) d x \geq \varphi_{i}\left(\widetilde{U}\left(\sigma_{i}(t)\right)\right) .
$$

Combining (9)-(12), we observe that $\widetilde{U}(t)$ is an eventually positive solution of (8) with $+\widetilde{G}(t)$. If $u<0$ in $G \times\left[t_{0}, \infty\right)$, we are also led to a contradiction by using the same arguments as in the case where $u>0$. The contradiction establishes the theorem.

\section{Higher Order Functional Differential Inequalities}

We derive sufficient conditions for the functional differential inequality

$$
\frac{d^{N}}{d t^{N}}\left(y(t)+\sum_{i=1}^{\ell} h_{i}(t) y\left(\rho_{i}(t)\right)\right)+\sum_{i=1}^{m} p_{i}(t) \varphi_{i}\left(y\left(\sigma_{i}(t)\right)\right) \leq q(t)
$$


to have no eventually positive solution, where $q(t) \in C\left(\left[t_{0}, \infty\right) ; \mathbb{R}\right)$ for some $t_{0}>0$.

In addition to the hypotheses $\left(\mathrm{H}_{1}\right)-\left(\mathrm{H}_{3}\right)$, we assume that:

$\left(\mathrm{H}_{4}\right) \quad N$ is an even positive integer;

$\left(\mathrm{H}_{5}\right) \quad \rho_{i}(t) \leq t(i=1,2, \ldots, \ell)$ and $\sum_{i=1}^{\ell} h_{i}(t) \leq 1$.

We use the following notation:

$$
[\Theta(t)]_{ \pm}=\max \{ \pm \Theta(t), 0\}
$$

for any continuous functions $\Theta(t)$.

Theorem 3: Assume that $\left(H_{1}\right)-\left(H_{3}\right)$ hold. If

$$
\liminf _{t \rightarrow \infty_{T}}^{t}\left(1-\frac{s}{t}\right)^{N-1} q(s) d s=-\infty
$$

for all large $T$, then inequality (13) has no eventually positive solution.

For the proof of this theorem see [6, Theorem 2] or [10, Lemma 3].

Theorem 4: Let $\left(H_{1}\right)-\left(H_{5}\right)$ hold. Assume that there exists a function $Q(t) \in$ $C^{N}\left(\left[t_{0}, \infty\right) ; \mathbb{R}\right)$ such that $Q^{(N)}(t)=q(t), Q(t)$ is oscillatory at $t=\infty$, and assume, moreover, that there exists an integer $j \in\{1,2, \ldots, m\}$ such that $\varphi_{j}(\xi)$ is nondecreasing for $\xi>0$. If

$$
\int^{\infty} p_{j}(s) \varphi_{j}\left(\left[\left(1-\sum_{i=1}^{\ell} h_{i}\left(\sigma_{j}(s)\right)\right) c+H\left(\sigma_{j}(s)\right)\right]_{+}\right) d s=\infty
$$

for any $c>0$, then (13) has no eventually positive solution, where

$$
H(t)=Q(t)-\sum_{i=1}^{\ell} h_{i}(t) Q\left(\rho_{i}(t)\right)
$$

Proof: Let $y(t)$ be an eventually positive solution of (13). Then $y(t)>0, y\left(\sigma_{i}(t)\right)>0$ $(i=1,2, \ldots, m), y\left(\rho_{i}(t)\right)>0(i=1,2, \ldots, \ell)$ on $\left[t_{1}, \infty\right)$ for some $t_{1} \geq t_{0}$. Since

$$
\frac{d^{N}}{d t^{N}}\left(y(t)+\sum_{i=1}^{\ell} h_{i}(t) y\left(\rho_{i}(t)\right)-Q(t)\right) \leq 0, t \geq t_{1}
$$

we find that $z^{(i)}(t) \quad(i=N-1, N-2, \ldots, 0)$ are monotone functions and either $z(t)>0$ or $z(t) \leq 0$ eventually, where

$$
z(t)=y(t)+\sum_{i=1}^{\ell} h_{i}(t) y\left(\rho_{i}(t)\right)-Q(t)
$$

If $z(t) \leq 0$ eventually, then we obtain

$$
0<y(t)+\sum_{i=1}^{\ell} h_{i}(t) y\left(\rho_{i}(t)\right) \leq Q(t)
$$


Since $Q(t)$ is oscillatory at $t=\infty$, the above inequality cannot be valid. Hence, $z(t)>0$ must hold eventually. From a result of Kiguradze [1], it follows that there exists an odd integer $k(0 \leq k \leq N)$ and a number $t_{2} \geq t_{1}$ such that

$$
\begin{gathered}
z^{(i)}(t)>0(0 \leq i \leq k), t \geq t_{2}, \\
(-1)^{i-k} z^{(i)}(t)>0(k \leq i \leq N), t \geq t_{2} .
\end{gathered}
$$

Therefore, we see that

$$
z^{\prime}(t)>0, z^{(N-1)}(t)>0, t \geq t_{2} .
$$

Using the inequality

$$
y(t) \leq z(t)+Q(t), \quad t>t_{2},
$$

we have

$$
\begin{gathered}
y(t)=z(t)-\sum_{i=1}^{\ell} h_{i}(t) y\left(\rho_{i}(t)\right)+Q(t) \\
\geq z(t)-\sum_{i=1}^{\ell} h_{i}(t)\left[z\left(\rho_{i}(t)\right)+Q\left(\rho_{i}(t)\right)\right]+Q(t) \\
\geq\left(1-\sum_{i=1}^{\ell} h_{i}(t)\right) z(t)+Q(t)-\sum_{i=1}^{\ell} h_{i}(t) Q\left(\rho_{i}(t)\right) \\
\geq\left(1-\sum_{i=1}^{\ell} h_{i}(t)\right) z\left(t_{3}\right)+H(t), t \geq t_{3}
\end{gathered}
$$

for some $t_{3} \geq t_{2}$, in view of the fact that $z(t)$ is an increasing function. Since $y(t)$ is positive, we obtain

$$
y(t) \geq\left[\left(1-\sum_{i=1}^{\ell} h_{i}(t)\right) z\left(t_{3}\right)+H(t)\right]_{+}, t \geq t_{3},
$$

and hence

$$
y\left(\sigma_{j}(t)\right) \geq\left[\left(1-\sum_{i=1}^{\ell} h_{i}\left(\sigma_{j}(t)\right)\right) z\left(t_{3}\right)+H\left(\sigma_{j}(t)\right)\right]_{+}, t \geq t_{4}
$$

for some $t_{4} \geq t_{3}$. Combining (13) with (15) yields 


$$
z^{(N)}(t)+p_{j}(t) \varphi_{j}\left(\left[\left(1-\sum_{i=1}^{\ell} h_{i}\left(\sigma_{j}(t)\right)\right) z\left(t_{3}\right)+H\left(\sigma_{j}(t)\right)\right]_{+}\right) \leq 0
$$

for $t \geq t_{4}$. Integrating (16) over $\left[t_{4}, t\right]$, we obtain

$z^{(N-1)}(t)-z^{(N-1)}\left(t_{4}\right)$

$$
+\int_{t_{4}}^{t} p_{j}(s) \varphi_{j}\left(\left[\left(1-\sum_{i=1}^{\ell} h_{i}\left(\sigma_{j}(s)\right)\right) z\left(t_{3}\right)+H\left(\sigma_{j}(s)\right)\right]_{+}\right) d s \leq 0
$$

and consequently,

$$
\begin{gathered}
\int_{t_{4}}^{t} p_{j}(s) \varphi_{j}\left(\left[\left(1-\sum_{i=1}^{\ell} h_{i}\left(\sigma_{j}(s)\right)\right) z\left(t_{3}\right)+H\left(\sigma_{j}(s)\right)\right]_{+}\right) d s \\
\leq-z^{(N-1)}(t)+z^{(N-1)}\left(t_{4}\right) \\
\leq z^{(N-1)}\left(t_{4}\right) .
\end{gathered}
$$

This contradicts the condition (14) and completes the proof.

\section{Oscillation of Partial Functional-Differential Equations}

In this section we establish oscillation criteria for the boundary value problems (1), ( $\left.\mathrm{B}_{i}\right)$ $(i=1,2)$ by combining the results of Sections 2 and 3.

Theorem 5: Assume that $\left(H_{1}\right)-\left(H_{3}\right)$ hold. If

$$
\liminf _{t \rightarrow \infty_{T}^{t}}^{t}\left(1-\frac{s}{t}\right)^{N-1} G(s) d s=-\infty
$$

and

$$
\limsup _{t \rightarrow \infty} \int^{t}\left(1-\frac{s}{T}\right)^{N-1} G(s) d s=\infty
$$

for all large $T$, then every solution $u$ of the boundary value problem (1), $\left(B_{1}\right)$ is oscillatory in $\Omega$.

Proof: The hypotheses imply that the functional differential inequalities 


$$
\frac{d^{N}}{d t^{N}}\left(y(t)+\sum_{i=1}^{\ell} h_{i}(t) y\left(\rho_{i}(t)\right)\right)+\sum_{i=1}^{m} p_{i}(t) \varphi_{i}\left(y\left(\sigma_{i}(t)\right)\right) \leq \pm G(t)
$$

have no eventually positive solutions. Hence, the inequality (2) also have no eventually positive solutions. The conclusion follows from Theorem 1.

Theorem 6: Assume $\left(H_{1}\right)-\left(H_{3}\right)$ hold. If

$$
\liminf _{t \rightarrow \infty_{T}^{t}}^{t}\left(1-\frac{s}{t}\right)^{N-1} \widetilde{G}(s) d s=-\infty
$$

and

$$
\limsup _{t \rightarrow \infty T} \int^{t}\left(1-\frac{s}{t}\right)^{N-1} \widetilde{G}(s) d s=\infty
$$

for all large $T$, then every solution $u$ of the boundary value problem $(1),\left(B_{2}\right)$ is oscillatory in $\Omega$.

Proof: The conclusion follows from Theorems 2 and 3.

Theorem 7: Let $\left(H_{1}\right)-\left(H_{5}\right)$ be satisfied. Assume that there exists a function $\Theta(t) \in C^{N}((0, \infty) ; \mathbb{R})$ such that $\Theta^{(N)}(t)=G(t)\left[\right.$ resp. $\left.\Theta^{(N)}(t)=\widetilde{G}(t)\right], \Theta(t)$ is oscillatory at $t=\infty$, and assume, moreover, that there exists an integer $j \in\{1,2, \ldots, m\}$ such that $\varphi_{j}(\xi)$ is nondecreasing for $\xi>0$. If

$$
\int^{\infty} p_{j}(s) \varphi_{j}\left(\left[\left(1-\sum_{i=1}^{\ell} h_{i}\left(\sigma_{j}(s)\right)\right) c+\widehat{\Theta}\left(\sigma_{j}(s)\right)\right]_{+}\right) d s=\infty
$$

and

$$
\int^{\infty} p_{j}(s) \varphi_{j}\left(-\left[\left(1-\sum_{i=1}^{\ell} h_{i}\left(\sigma_{j}(s)\right)\right) c-\widehat{\Theta}\left(\sigma_{j}(s)\right)\right]_{+}\right) d s=-\infty
$$

for any $c>0$, then every solution $u$ of the boundary problem (1), $\left(B_{1}\right)\left[\right.$ resp. $\left.(1),\left(B_{2}\right)\right]$ is oscillatory in $\Omega$, where

$$
\widehat{\Theta}(t)=\Theta(t)-\sum_{i=1}^{\ell} h_{i}(t) \Theta\left(\rho_{i}(t)\right)
$$

Proof: The conclusion follows by combining Theorem 4 with Theorems 1 and 2 .

Theorem 8: Let $\left(H_{1}\right)-\left(H_{5}\right)$ be satisfied. Assume that there exists a function $\Theta(t) \in C^{N}((0, \infty) ; \mathbb{R})$ such that $\Theta^{(N)}(t)=G(t), \Theta(t)$ is oscillatory at $t=\infty$. If

$$
\int^{\infty}\left(\sum_{j=1}^{M} \lambda_{1}^{j} a_{j}(t)\right)\left[\left(1-\sum_{i=1}^{\ell} h_{i}\left(\sigma_{j}(s)\right)\right) c \pm \widehat{\Theta}\left(\sigma_{j}(s)\right)\right]_{+} d s=\infty
$$


for any $c>0$, then every solution $u$ of the boundary value problem $(1),\left(B_{1}\right)$ is oscillatory in $\Omega$.

Proof: Theorem 4 implies that

$$
\frac{d^{N}}{d t^{N}}\left(y(t)+\sum_{i=1}^{\ell} h_{i}(t) y\left(\rho_{i}(t)\right)\right)+\left(\sum_{j=1}^{M} \lambda_{1}^{j} a_{j}(t)\right) y(t) \leq \pm G(t)
$$

have no eventually positive solutions. Hence, the inequalities (2) have no eventually positive solutions. The conclusion follows from Theorem 1.

Remark 1: Theorem 5 was obtained by Yoshida [10].

Remark 2: When $N=2$, Theorem 7 was established by Tanaka [7].

Remark 3: In the case where $M=1, f(x, t) \equiv 0, \psi_{1}=\widetilde{\psi}_{1} \equiv 0$, Theorem 7 reduces to a result of Liu and $\mathrm{Fu}[4]$.

Example 1: We consider the problem

$$
\begin{gathered}
\frac{\partial^{4}}{\partial t^{4}}\left(u(x, t)+\frac{1}{2} u(x, t-\pi)\right)-\frac{\partial^{2} u}{\partial x^{2}}(x, t)+\frac{1}{2} \frac{\partial^{4} u}{\partial x^{4}}(x, t) \\
+u(x, t-2 \pi)=3 \sin x \sin t, \quad(x, t) \in(0, \pi) \times(0, \infty), \\
u(0, t)=u(\pi, t)=\frac{\partial^{2} u}{\partial x^{2}}(0, t)=\frac{\partial^{2} u}{\partial x^{2}}(\pi, t)=0, t>0 .
\end{gathered}
$$

Here $n=1, G=(0, \pi), \Omega=(0, \pi) \times(0, \infty), N=4, \ell=1, h_{1}(t)=1 / 2, \rho_{1}(t)=t-\pi$, $M=2, a_{1}(t)=1, a_{2}(t)=1 / 2, b_{i j}(t) \equiv 0(i=1,2, \ldots, k ; j=1,2), m=1, p_{1}(t)=1$, $\varphi_{1}(\xi)=\xi, \sigma_{1}(t)=t-2 \pi, f(x, t)=3 \sin x \sin t$ and $\psi_{1}=\psi_{2} \equiv 0$. It is easy to see that $\lambda_{1}=1, \Phi(x)=\sin x, \Psi_{1}(t)=\Psi_{2}(t) \equiv 0$ and $G(t)=F(t)=(3 / 4) \pi \sin t$. Choosing $\Theta(t)=(3 / 4) \pi \sin t$, we observe that $\Theta^{(4)}(t)=G(t)$ and $\Theta(t)$ is oscillatory at $t=\infty$. Furthermore, we find that $\widehat{\Theta}(t)=\widehat{\Theta}(t-2 \pi)=(9 / 8) \pi \sin t$. A simple calculation yields

$$
\int^{\infty}\left[\frac{1}{2} c \pm \frac{9}{8} \pi \sin s\right]_{+} d s=\infty
$$

for any $c>0$. Hence, it follows from Theorem 7 that every solution $u$ of the problem (17), (18) is oscillatory in $(0, \pi) \times(0, \infty)$. Indeed, $u=\sin x \sin t$ is such a solution.

Example 2: We consider the problem

$$
\begin{gathered}
\frac{\partial^{6}}{\partial t^{6}}\left(u(x, t)+\frac{1}{3} u(x, t-(\pi / 2))\right)-\frac{\partial^{2} u}{\partial x^{2}}(x, t)+\frac{\partial^{4} u}{\partial x^{4}}(x, t) \\
+\frac{1}{3} u(x, t-(\pi / 2))=\cos x \sin t, \quad(x, t) \in(0, \pi) \times(0, \infty), \\
-\frac{\partial u}{\partial x}(0, t)=\frac{\partial u}{\partial x}(\pi, t)=-\frac{\partial^{3} u}{\partial x^{3}}(0, t)=\frac{\partial^{3} u}{\partial x^{3}}(\pi, t)=0, \quad t>0 .
\end{gathered}
$$

Here $n=1, G=(0, \pi), \Omega=(0, \pi) \times(0, \infty), N=6, \ell=1, h_{1}(t)=1 / 3, \rho_{1}(t)=$ $t-(\pi / 2), \quad M=2, \quad a_{1}(t)=a_{2}(t)=1, \quad b_{i j}(t) \equiv 0 \quad(i=1,2, \ldots, k ; \quad j=1,2), \quad m=1$, $p_{1}(t)=1 / 3, \varphi_{1}(\xi)=\xi, \sigma_{1}(t)=t-(\pi / 2), f(x, t)=\cos x \sin t, \mu_{1}=\mu_{2} \equiv 0$ and $\widetilde{\psi}_{1}=$ $\widetilde{\psi}_{2} \equiv 0$. It is easily checked that $\widetilde{\Psi}_{1}(t)=\widetilde{\Psi}_{2}(t) \equiv 0$ and $\widetilde{G}(t)=\widetilde{F}(t) \equiv 0$. We can 
choose $\Theta(t) \equiv 0$, and observe that $\Theta^{(6)}(t)=\widetilde{G}(t), \Theta(t)$ is oscillatory at $t=\infty$ and $\widehat{\Theta}(t) \equiv 0$. An easy computation shows that

$$
\int^{\infty} \frac{1}{3}\left[\left(1-\frac{1}{3}\right) c\right]_{+} d s=\infty
$$

for any $c>0$. Therefore, Theorem 7 implies that every solution $u$ of the problem (19), (20) is oscillatory in $(0, \pi) \times(0, \infty)$. One such solution is $u=\cos x \sin t$.

\section{References}

[1] Kiguradze, I.T., On the oscillation of solutions of the equation $\frac{d^{m} u}{d t^{m}}+a(t)|u|^{n}$ sign $u=0$, Mat. Sb. 65 (1964), 172-187.

[2] Kiguradze, T. and Stavroulakis, I.P., On oscillatory properties of solutions of higher order linear hyperbolic equations, Adv. Math. Sci. Appl. (to appear).

[3] Kusano, T. and Yoshida, N., Oscillation criteria for a class of nonlinear partial differential equations, J. Math. Anal. Appl. 79 (1981), 236-243.

[4] Liu, X. and Fu, X., Oscillation criteria for high order delay partial differential equations, J. Appl. Math. Stochastic Anal. 11 (1998), 193-208.

[5] Onose, H. and Yokoyama, E., Oscillation of partial differential inequalities, Tamkang J. Math. 7 (1976), 67-70.

[6] \# \#ana, P., Oscillation criteria for forced nonlinear elliptic equations of arbitrary order, \#

[7] Tanaka, S., Oscillation properties of solutions of second order neutral differential equations with deviating arguments, Analysis 17 (1997), 99-111.

[8] Travis, C.C. and Yoshida, N., Oscillation criteria for nonlinear Bianchi equations, Nonlinear Anal. 6 (1982), 625-636.

[9] Yoshida, N., Forced oscillations of extensible beams, SIAM J. Math. Anal. 16 (1985), 211-220.

[10] Yoshida, N., Oscillation of partial functional-differential equations with deviating arguments, Tamkang J. Math. 26 (1995), 131-139. 


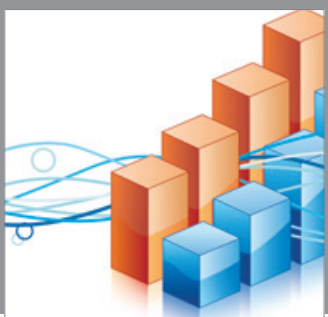

Advances in

Operations Research

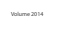

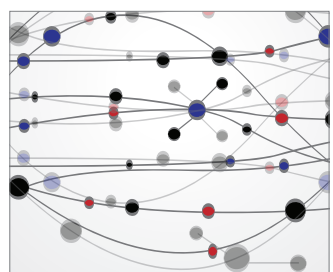

\section{The Scientific} World Journal
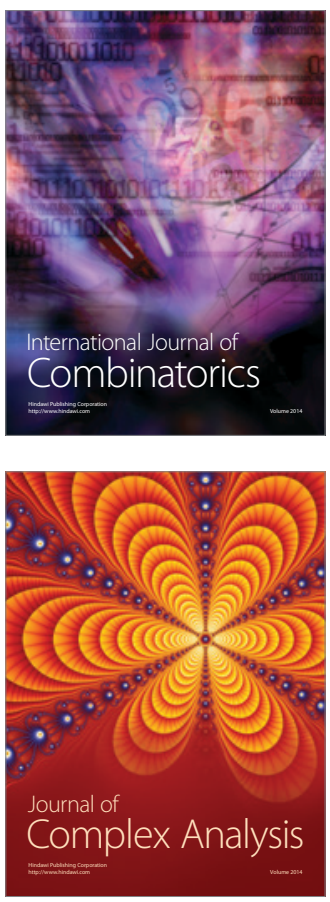

International Journal of

Mathematics and

Mathematical

Sciences
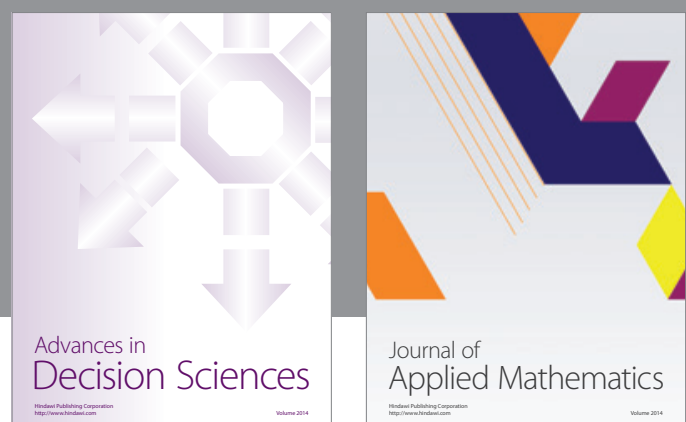

Journal of

Applied Mathematics
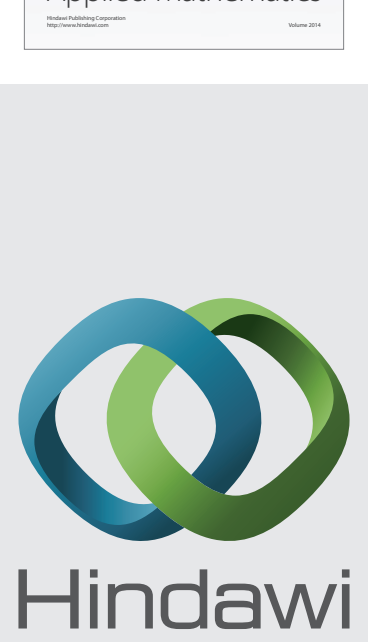

Submit your manuscripts at http://www.hindawi.com
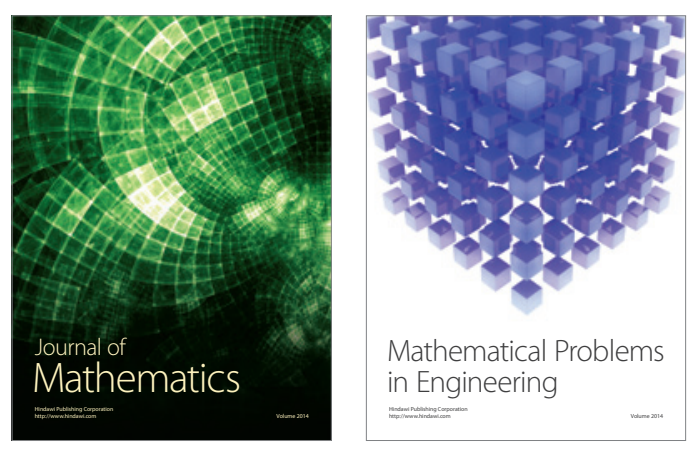

Mathematical Problems in Engineering
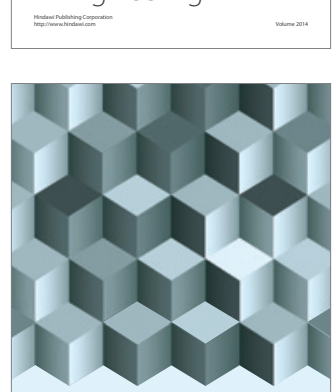

Journal of

Function Spaces
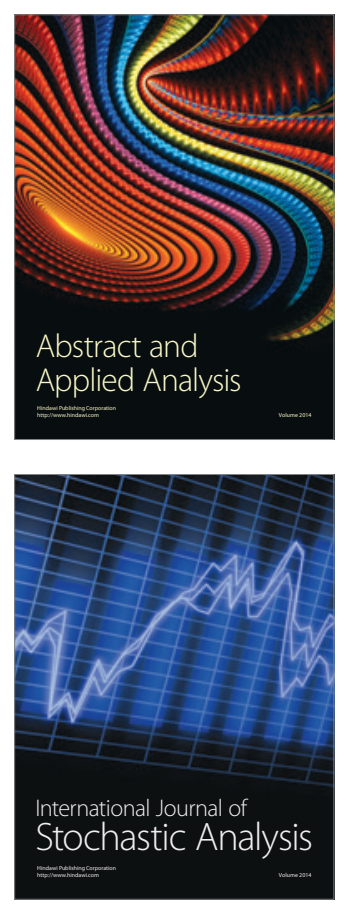

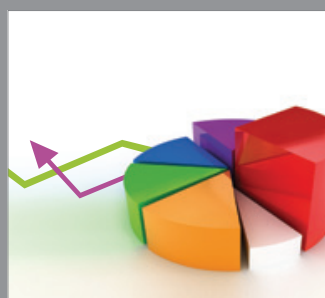

ournal of

Probability and Statistics

Promensencen
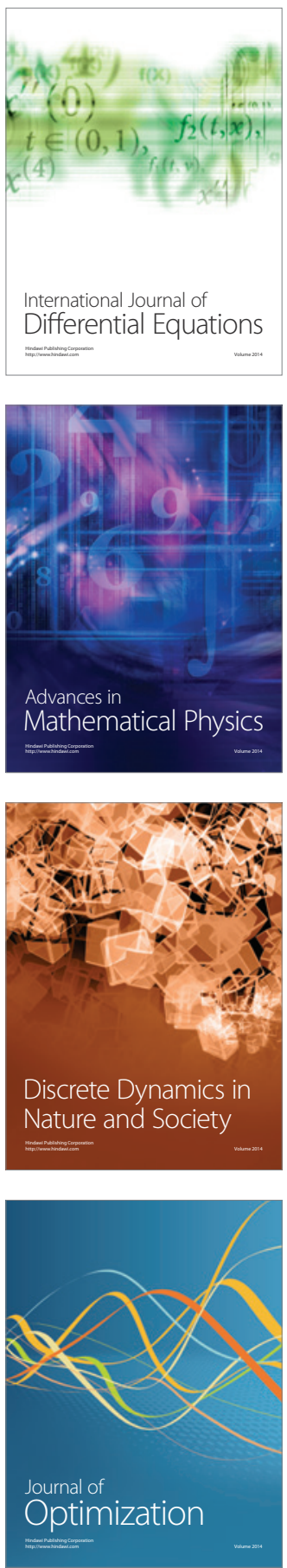